Methods All patients commenced on rifaximin for HE from $1^{\text {st }}$ January to $31^{\text {st }}$ December 2011 were identified from the records of the pharmacy department at Freeman Hospital. The number and length of emergency hospital admissions for the period 1 year prior to starting rifaximin was compared to 1 year after starting the drug. Cost effectiveness was calculated using the standard British National Formulary (BNF) tariff for rifaximin and the estimated cost per day for acute inpatient admission to the Newcastle upon Tyne Hospitals Trust.

Results 64 patients (75\% male, 53\% ALD) were identified, 40 $(63 \%)$ were on concomitant lactulose. In 8 patients rifaximin was discontinued ( 5 after transplant and 3 when HE excluded). 23 (36\%) patients died within 1 year (median survival 62 days (range 2-364) and $33(52 \%)$ were alive at 1 year and remained on rifaximin. Mean MELD of survivors was significantly lower than non-survivors (13.0 vs. $19.0 \mathrm{P}<0.05$ ) and scores predicted 28 day mortality (median MELD 29 (range 4-37)). Complete data were available for 25 of the survivors and showed a significant reduction in the number of emergency admissions from a mean of 2.8 to 1.7 admissions per patient per year with rifaximin $(\mathrm{P}<0.05)$. Duration of inpatient admission decreased significantly from a mean of 30.2 to 9.8 bed days per patient per year $(\mathrm{P}<0.05)$. Taking into account the cost of one year's treatment with rifaximin ( $£ 3,687 \mathrm{GBP})$ the reduction in the number of emergency admissions represents an annual saving of $£ 3,468 \mathrm{GBP}$ per patient.

Conclusion Treatment with rifaximin for secondary prevention of hepatic encephalopathy appears to be cost effective at reducing emergency admission to hospital.

Disclosure of Interest None Declared

\section{PTU-113 RIFAXIMIN IN NON-ALCOHOLIC STEATOHEPATITIS: AN OPEN-LABEL PILOT STUDY}

doi:10.1136/gutjnl-2013-304907.203

1."J F Cobbold, 'S N Wai, 'J Stove, 'F Shojaee-Moradie, 'J Fitzpatrick, 'E L Thomas, 1J Bell, ' $R$ D Goldin, ' S D Taylor-Robinson, 'M S Yee, ${ }^{3} \mathrm{Q}$ M Anstee, 'M R Thursz. 'Imperial College London, London; 'University of Surrey, Guildford; ' $U$ niversity of Newcastle, Newcastle-upon-Tyne, UK

Introduction Mounting evidence implicates gut microbial dysbiosis in the pathogenesis of non-alcoholic steatohepatitis (NASH) by mechanisms including caloric salvage, lipopolysaccharide production, upregulation of proinflammatory cytokines, increased insulin resistance and consequent increases in body mass and hepatic steatosis. Rifaximin is a minimally-absorbed, gut-selective antibiotic with bactericidal activity against a broad spectrum of gut microbes, making it an attractive candidate therapy. We aimed to study the effect of Rifaximin on markers of hepatic inflammation, hepatic steatosis, hepatic and peripheral insulin sensitivity.

Methods Patients with biopsy-proven NASH, elevated aminotransferase values and no hepatic comorbidities were included in this open-label, randomised, cross-over study, all receiving 6 weeks of Rifaximin $400 \mathrm{mg}$ twice daily, before or after one of two 6 week observation periods on standard therapy. The primary endpoint was change in alanine aminotransferase (ALT) values after Rifaximin therapy. Secondary endpoints were change in percentage hepatic lipid assessed by hepatic proton magnetic resonance spectroscopy and change in hepatic and peripheral insulin sensitivity assessed by the hyperinsulinaemic euglycaemic clamp. Patients also had anthropometrics, serum biochemistry and cytokine profiling at each timepoint. Stool and urine were collected for subsequent analysis.

Results 15 patients, 13 male, 2 female, mean (SD) age 48(9.5) years were included. 7 had diabetes on oral hypoglycaemic medications and 8 did not have diabetes. After 6 weeks of therapy, there was no difference in ALT before, 69 (40)IU/L, and after, 71 (43)IU/L, treatment, $\mathrm{p}=0.7$. Hepatic lipid content was $23.3(12.8) \%$ before and 26.5(15.9)\% after Rifaximin, $p=0.16$. Peripheral insulin sensitivity (Rd) was unchanged, 29.5 (6.5) to $29.4(10.0) \mathrm{mmol} / \mathrm{kg}$ min, $p=0.91$, hepatic insulin sensitivity (\% suppression of endogenous glucose production) was unchanged $(35.2(10.1) \%$ to $31.2(13.2) \%, p=0.35)$. There were no significant differences in body mass index, waist and hip circumference, IL 1b, IL6, IL10, IL18, CD14, TNFa, Leptin, Resistin and Adiponectin values with treatment.

Conclusion Treatment with Rifaximin was not associated with changes in markers of hepatocellular damage, hepatic lipid content, cytokine profile or insulin sensitivity in patients with NASH.

Disclosure of Interest None Declared

\section{PTU-114 SEROLOGICAL AUTOANTIBODIES IN HEALTH AND LIVER DISEASE IN A BLACK AFRICAN POPULATION}

doi:10.1136/gutjnl-2013-304907.204

1,"J A Otegbayo, ' G 0 Arinola. 'medicine, University College Hospital, Ibadan, Nigeria

Introduction Some pathologic autoantibodies are useful in the diagnosis of autoimmune liver diseases. There is dearth of literature on the prevalence and pattern of autoantibodies in black population of Africans with liver disease, and none from Nigeria. This study sought to determine the prevalence and pattern of autoantibodies among patients with liver diseases and apparently healthy individuals in Nigeria.

Methods The seroprevalence of antinuclear antibodies (ANA), antimitochondrial antibodies (AMA), anti-liver kidney microsomal antibodies (Anti-LKM-1), anti-soluble liver antigen/liver pancreas (Anti-SLA-LP), perinuclear anti-neutrophil cytoplasmic antibodies (pANCA), were analysed in patients with liver diseases and apparently health controls, using ELISA method. Appropriate statistical methods were used for Odds ratio, Pearson Chi square and students' t-test. Significant statistical difference was specified at $\mathrm{p}<0.05$

Results One hundred and twenty six patients with liver diseases (91 (72.2\%) males and $35(27.8 \%)$ females) and 82 apparently normal control subjects (59 (72\%) males and 23 (28\%) females) were studied over a two year period. The patients consisted of HCC 77 (61.1\%), liver cirrhosis 32 (25.4\%), chronic hepatitis $10(7.9 \%)$, acute viral hepatitis $4(3.2 \%)$, alcoholic cirrhosis $1(0.8 \%)$ and primary biliary cirrhosis $2(1.6 \%)$.

The control group consisted of Eighty two (82) apparently normal individuals consisting of were recruited over the study period to serve as controls. Of the 126 cases and 82 controls analysed for autoantibodies, except for ANA ( 107 cases and 67 controls) for autoantibodies only AMA was found to be significantly higher among cases compared with controls. Antimitochondrial antibodies were present in $76(60.3 \%)$ of the cases compared with $36(43.9 \%)$ controls $(p<0.05)$, while ANA were present in $42(39.3 \%)$ of cases compared with $27(39.7 \%)$ controls $(p=0.68)$. Anti-soluble liver antigen (anti-SLA/LP) and pANCA were completely absent among cases and controls Table 2 .

Chronic hepatitis had the highest frequency of AMA, being positive in $9(90 \%)$ of the 10 cases, this was followed by HCC, $48(62.3 \%)$ of the 77 cases tested were positive for AMA.

Conclusion Serological autoantibodies were equally present in both liver diseases and in health, and would not be sufficient for the diagnosis of autoimmune liver disease in Africans. Therefore, other parameters have to be considered whenever there is a clinical suspicion of autoimmune liver disease among Nigerians.

Disclosure of Interest None Declared 